\title{
NÓDULOS DE SCHMORL EN RESTOS HUMANOS ARQUEOLÓGICOS DE PATAGONIA AUSTRAL
}

JORGE A. SUBY

\section{RESUMEN}

Los nódulos de Schmorl (NS) son herniaciones del núcleo pulposo de los discos intervertebrales dentro de los cuerpos vertebrales adyacentes. Las causas de su desarrollo son parcialmente comprendidas, asociadas a factores morfológicos, degenerativos, traumáticos y metabólicos, mientras el rol de su interpretación como indicador de actividad física es aún cuestionado. En este trabajo se estudia la prevalencia de NS en 22 esqueletos arqueológicos de Patagonia Austral, discutiendo sus posibles causas y relación con los estilos de vida propuestos para las poblaciones humanas de esa región. Se registró una prevalencia de 27,3\% para el total de los esqueletos analizados. Además, se encontró una mayor prevalencia de NS en la región de Santa Cruz/Magallanes en relación a los esqueletos de Tierra del Fuego. Aunque no puede descartarse una tendencia biológica al desarrollo de estas lesiones, los resultados sugieren un mayor grado de estrés mecánico sobre la columna vertebral en los individuos de la región continental de Patagonia Austral.

PALABRAS CLAVE: nódulos de Schmorl, restos humanos arqueológicos, Patagonia austral, paleopatología

\section{SCHMORL'S NODES IN ARCHAEOLOGICAL SKELETAL REMAINS FROM SOUTHERN PATAGONIA}

\begin{abstract}
ABTRACT
Schmorl's Nodes are herniations of the intervertebral disc nucleous pulposus into the adjacent vertebra. Their causes are partially understood and associated to morphological, degenerative, traumatic and metabolic factors, whereas their role as an indicator of physical activity is currently under debate. In this paper the prevalence of NS in 22 archaeological skeletons from Southern Patagonia is studied, discussing their possible causes and their relationship with the lifestyles of the human populations of this region. A prevalence of $27.3 \%$ was recorded. The skeletons from the Santa Cruz/Magallanes region showed higher prevalence of NS than skeletons from northern and southern Tierra del Fuego. Although biological susceptibility for this difference could not be

CONICET - INCUAPA - Laboratorio de Ecología Evolutiva Humana, Departamento de Arqueología, Unidad de Enseñanza Académica Quequén, Universidad Nacional del Centro de la Provincia de Buenos Aires. Calle 508 Nro. 881. CP 7631, Quequén, Buenos Aires, Argentina. E-mail: jasuby@conicet.gov.ar/jasuby@gmail.com.
\end{abstract}


discarded, the results suggest that high mechanical stress on the vertebras is responsible for the developed NS in continental Southern Patagonia.

KEYWORDS: Schmorl's nodes, archaeological human remains, Southern Patagonia, paleopathology

\section{INTRODUCCIÓN}

Los nódulos de Schmorl (NS) son herniaciones del núcleo pulposo de los discos intervertebrales dentro de los cuerpos vertebrales adyacentes (Pfirrmann \& Resnick 2001; Wagner et al. 2000; Zhang et al. 2010). El término es adaptado en el caso de los estudios de restos humanos esqueletales para designar la lesión ósea final sobre la superficie del cuerpo vertebral, caracterizada morfológicamente como un defecto redondeado o en forma de riñón, con márgenes escleróticos suaves o rugosos (Faccia \& Williams 2008; Novak \& Šlaus 2011; Williams et al. 2007).

Aunque el mecanismo por el cual se producen los NS no son completamente comprendidos (Plomp et al. 2012; Zhang et al. 2010), la teoría original y más aceptada actualmente (Schmorl \& Junghans 1971) sugiere que son el resultado del debilitamiento de los platillos vertebrales, los cuales tienden a ser más delgados en la región central adyacente al núcleo pulposo (Moore 2006; Plomp et al. 2012; Roberts et al. 1989). Los discos intervertebrales son estructuras cilíndricas, compuestas por un núcleo pulposo central altamente hidratado, rodeado por un anillo fibroso de colágeno, firme pero flexible (Fig.1) (Yasuma et al. 1988). En la porción craneal y caudal de cada disco se encuentran los platillos vertebrales, compuestos por tejido cartilaginoso, que separan las vértebras de los discos (Moore 2006). Los platillos vertebrales, de aproximadamente 1 $\mathrm{mm}$ de espesor, absorben la presión hidrostática que resulta de la carga mecánica producida sobre la columna vertebral y desempeñan un papel central en el metabolismo del disco intervertebral gracias a la activa comunicación a través de vasos sanguíneos entre ambos (Moore 2006). En condiciones normales, los platillos vertebrales previenen que el núcleo pulposo penetre en las vértebras adyacentes, impidiendo así la formación de NS. Sin embargo, debido a estas áreas de debilitamiento los platillos vertebrales no resisten adecuadamente la presión, lo que produce el prolapso del núcleo dentro de la vértebra a través del platillo (Moore 2006; Schmorl \& Junghans 1971).

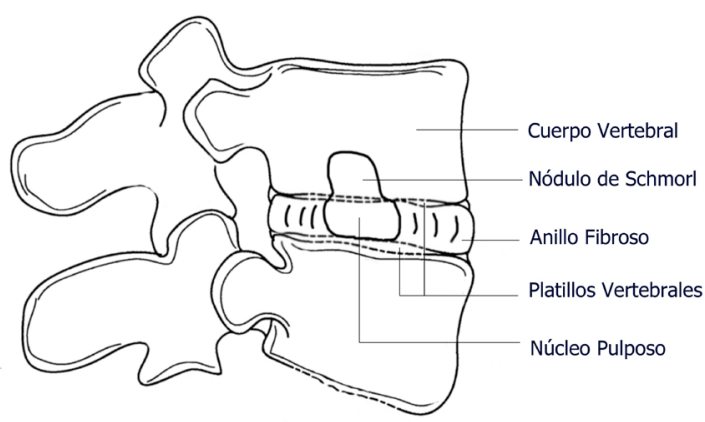

Fig. 1. Estructura anatómica básica de los discos intervertebrales, platillos vertebrales y nódulos de Schmorl.

Entre las causas de formación de los NS se han propuesto procesos degenerativos, traumáticos y metabólicos (González-Reimers et al. 2002; Fahey et al. 1998; Pfirrmann \& Resnick 2001; Zhang et al. 2010). De acuerdo con Schmorl \& Junghans (1971), los NS pueden estar relacionados con cambios degenerativos articulares asociados a daños por fatiga y estrés sobre la columna vertebral. Algunos autores sugieren una relación entre los NS y la presencia de osteofitos de moderado desarrollo (Fahey et al. 1998; Pfirrmann \& Resnick 2001), mientras otras investigaciones no encuentran tal asociación (González-Reimers et al. 2002). Las fracturas de los platillos vertebrales por traumas agudos pueden también causar rupturas de los discos intervertebrales, facilitando el desarrollo de NS (Faccia \& Williams 2008; Fahey et al. 1998; Wagner et al. 2000). Trastornos metabólicos como la osteoporosis han sido expuestos como posibles factores favorecedores de los NS, al producir debilitamiento del tejido óseo subcondral de los cuerpos vertebrales, aunque esta asociación permanece en discusión (González-Reimers et al. 2002; Pfirrmann \& Resnick 2001).

Otras causas fueron sugeridas, incluyendo la posible influencia de áreas de debilitamiento 
congénitas de los platillos vertebrales, producidas como consecuencia de la regresión de la notocorda durante etapas embrionarias (Schmorl \& Junghans 1971), lo que podría explicar la alta frecuencia observada en individuos jóvenes (Dar et al. 2010). Resultados recientes proponen que este debilitamiento estaría relacionado con cambios en los patrones vasculares entre el disco y el platillo vertebral (Haidar et al. 2009) o con necrosis isquémicas óseas subcondrales producidas debajo del platillo vertebral (Peng et al. 2003). Además, Zhang et al. (2010) plantean como hipótesis un rol destacado de causas inmunológicas en el desarrollo de los NS.

Los NS son probablemente los defectos más comunes de los platillos vertebrales, por lo que constituyen hallazgos frecuentes. En poblaciones modernas se observó un amplia variabilidad en la prevalencia de los NS, estimada entre 30\% y $75 \%$ (Pfirrmann \& Resnick 2001; Resnick \& Niwayama 1978; Schmorl \& Junghanns 1971; Hilton et al. 1976), dependiendo en gran medida de los métodos de registro empleados, radiográficos y tomográficos en individuos vivos, o a partir de análisis de cuerpos provenientes de autopsias o restos esqueletales, y de si son considerados casos sintomáticos o asintomáticos (Dar et al. 2010). Los estudios de la prevalencia de NS en poblaciones antiguas acompañan en general esta variabilidad de las frecuencias (Üstündağ 2009).

Usualmente los NS afectan el segmento de las últimas vértebras dorsales y primeras vértebras lumbares (Dar et al. 2010; Pfirrmann \& Resnick 2001). Algunos autores reportan una mayor prevalencia en hombres (Pfirrmann \& Resnick 2001), mientras otros contradicen estas tendencias sexuales. Por ejemplo, Dar et al. (2010), en un estudio sobre una extensa muestra de restos óseos de la colección Hamman-Todd, no hallaron diferencias entre sexos.

De acuerdo a su ubicación, se reconocen NS centrales y periféricos (Chandraraj et al. 1998). Se han reportado mayores frecuencias en regiones centrales de las vértebras, seguidas por lesiones posteriores en las cuales el material nuclear se extruye hacia el foramen intervertebral y/o la médula espinal. Por el contrario, en baja frecuencia afectan la superficie anterior de los platillos vertebrales (Dar et al. 2010; Faccia \& Williams 2008; Pfirrmann \&
Resnick 2001). Las hernias centrales es el tipo que ha sido relacionado con mayores síntomas, y por lo tanto más comúnmente diagnosticado en casos clínicos (Chandraraj et al. 1998; Faccia \& Williams 2008), aunque su asociación con el dolor es motivo de discusión (Takahashi et al. 1995).

A pesar de la alta frecuencia de hallazgos en restos esqueletales, existen sólo algunos estudios paleopatológicos centrados en el estudio de los NS (Lai \& Lovell 1992; McNaught 2006; Robb et al. 2001; Saluja et al. 1986; Šlaus 2000; Üstündağ 2009; Weiss 2005). De manera similar a otras regiones, hasta el momento se desconoce la prevalencia de NS en poblaciones cazadorasrecolectoras del sur de Sudamérica. Por este motivo, el objetivo de este trabajo es estudiar la presencia y prevalencia de NS en restos óseos humanos arqueológicos de Patagonia Austral, así como su distribución anatómica, sexo-etaria y regional que permitan discutir las posibles causas de su desarrollo y su relación con los estilos de vida propuestos para las poblaciones humanas de esta región.

\section{MATERIALES Y MÉTODOS}

Fueron estudiados esqueletos humanos recuperados en Patagonia Austral alojados en colecciones de museos e instituciones de investigación de Argentina (Museo del Fin del Mundo, Ushuaia; IMHICIHU, Buenos Aires; Museo Etnográfico J. B. Ambrosetti, Buenos Aires; CADIC, Ushuaia; y Laboratorio de Ecología Evolutiva Humana- UNCPBA, Quequén) y Chile (Instituto de la Patagonia, Punta Arenas). Se incluyeron sólo aquellos esqueletos adultos de ambos sexos con un alto porcentaje de los cuerpos vertebrales presentes y en buenas condiciones de preservación que permitan evaluar la presencia de NS, que además tuvieran información contextual espacio-temporal y paleodietaria a partir de análisis previos de isótopos estables.

En total fueron seleccionados 22 individuos provenientes de Patagonia Austral (Tabla 1, Fig. 2). Los individuos fueron posteriormente clasificados de acuerdo a su región de origen. Del total de esqueletos seleccionados, $8(36,4 \%)$ fueron recuperados en la costa de Santa Cruz/ Magallanes (región meridional continental), $8(36,4 \%)$ en el 
norte de Tierra del Fuego y $6(27,2 \%)$ en el sur de Tierra del Fuego (Tabla 1). De los esqueletos incluidos, $3(13,6 \%)$ corresponden a momentos previos a los ca. 3.500 años AP, $8(36,4 \%)$ al periodo ca. 1.500-600 años AP y 11 (50\%) a momentos históricos.

Para la determinación del sexo se tuvieron en cuenta las características dimórficas de la pelvis y el cráneo, siguiendo los criterios propuestos por Buikstra \& Ubelaker (1994). Del total de esqueletos estudiados, $5(22,7 \%)$ pertenecen a individuos femeninos y $17(77,3 \%)$ a individuos masculinos (Tabla 1). Se estimó la edad al momento de la muerte siguiendo los métodos de Brooks \& Suchey (1990), Todd (1921, en Buikstra and Ubelaker, 1994) e Isçan et al. (1984, en Bass 1995). La muestra incluye 11 (50\%) individuos adultos jóvenes entre 18-34 años y 11 (50\%) adultos medios entre 35-49 años (Tabla 1). Los resultados de isotopos estables previamente informados indican que 10 esqueletos $(45,4 \%)$ presentan una dieta predominantemente terrestre, 6 individuos $(27,3 \%)$ corresponden a dietas con mayor consumo de recursos marítimos y los restantes $6(27,3 \%)$ a dietas que pueden considerarse mixtas (Tabla 1), en el sentido propuesto por Borrero y Barberena (2006).

La superficie superior e inferior de los cuerpos de cada vértebra cervical, dorsal y lumbar fue inspeccionada macroscópicamente en busca de la presencia de NS. Se registró el número de NS por individuo, las vértebras involucradas, la superficie afectada (superior/inferior) y su ubicación en el cuerpo vertebral (tercio anterior/ central/posterior). Se registró además la severidad, estimada según el criterio propuesto por Knüsel et al. (1997), calificando como 1 (leve) a los NS de menos de $2 \mathrm{~mm}$ de profundidad y que cubren un área equivalente a menos de la mitad de la distancia anteroposterior del cuerpo vertebral, $y$

Tabla 1. Restos esqueletales e información asociada incluidos en este trabajo. Ref: $M=$ masculino; $F=$ femenino; $\mathrm{T}=$ terrestre; Mix=mixta; $\mathrm{Ma}=$ marítima. Los fechados radiocarbónicos se presentan sin calibrar. *: AA94279.

\begin{tabular}{|c|c|c|c|c|c|c|}
\hline Región & Sitio & Sexo & Edad & Cronología & Dieta & Referencia \\
\hline \multirow{8}{*}{$\begin{array}{l}\text { Santa Cruz/ } \\
\text { Magallanes } \\
n=8\end{array}$} & Juni Aike & M & $30-40$ & Histórico & $\mathrm{T}$ & Borrero \& Barberena 2006 \\
\hline & Puerto Natales 3 & $\mathrm{~F}$ & $20-25$ & Histórico & Mix & Guichón et al. 2006 \\
\hline & Cañadón Misionero & M & $35-45$ & $70 \pm 30$ & $\mathrm{~T}$ & Suby et al. 2009 \\
\hline & Rincón del Buque & M & $35-40$ & $830 \pm 42$ & $\mathrm{~T}$ & Suby et al. 2009 \\
\hline & Cabo Virgenes 17 & M & $20-34$ & $900 \pm 40$ & Mix & L'Heureux et al. 2003 \\
\hline & Punta Daniel & M & $30-40$ & $1118 \pm 43^{*}$ & Mix & Borrero \& Barberena 2006 \\
\hline & Orejas de Burro 1 & M & $20-25$ & $3565 \pm 45$ & $\mathrm{~T}$ & L'Heureux \& Barberena 2008 \\
\hline & Orejas de Burro 2 & M & $45-50$ & $3565 \pm 45$ & $\mathrm{~T}$ & L'Heureux \& Barberena 2008 \\
\hline \multirow{8}{*}{$\begin{array}{l}\text { Norte TdF } \\
n=8\end{array}$} & Las Mandíbulas & M & 24 & Histórico & $\mathrm{T}$ & Guichón et al. 2000 \\
\hline & Bahía Gente Grande & M & $30-40$ & Histórico & $\mathrm{T}$ & Schinder \& Guichón 2003 \\
\hline & Lengua de Vaca & $\mathrm{F}$ & $30-40$ & $251 \pm 41$ & Mix & Schinder \& Guichón 2003 \\
\hline & Puesto Pescador 1 & M & $21-25$ & $335 \pm 35$ & $\mathrm{~T}$ & Suby et al. 2008 \\
\hline & Estancia San Julio & M & $40-50$ & $350 \pm 50$ & $\mathrm{~T}$ & Suby \& Guichón 2010 \\
\hline & Myren 1 & M & $18-23$ & $640 \pm 20$ & Mix & Schinder \& Guichón 2003 \\
\hline & Bahía Felipe 1 & M & $35-50$ & $1608 \pm 45$ & Mix & Schinder \& Guichón 2003 \\
\hline & La Arcillosa 2 & $\mathrm{~F}$ & $20-24$ & $5208 \pm 58$ & $\mathrm{~T}$ & Santiago et al. 2011 \\
\hline \multirow{6}{*}{$\begin{array}{l}\text { Sur TdF } \\
n=6\end{array}$} & Acatushun & $\mathrm{F}$ & $30-40$ & Histórico & Ma & Piana et al. 2006 \\
\hline & Harberton Cementerio & M & $25-30$ & Histórico & $\mathrm{Ma}$ & Piana et al. 2006 \\
\hline & Bahia Tetys & M & $30-39$ & $450 \pm 60$ & $\mathrm{Ma}$ & Tessone et al. 2011 \\
\hline & Caleta Falsa 1 sitio 8 & M & $18-23$ & $820 \pm 40$ & $\mathrm{Ma}$ & Guichón \& Suby 2011 \\
\hline & Paiashauaia & $\mathrm{F}$ & $35-39$ & $1504 \pm 46$ & $\mathrm{Ma}$ & Piana et al. 2006 \\
\hline & Shamakush 6 & M & $35-39$ & $1536 \pm 46$ & $\mathrm{Ma}$ & Piana et al. 2006 \\
\hline
\end{tabular}




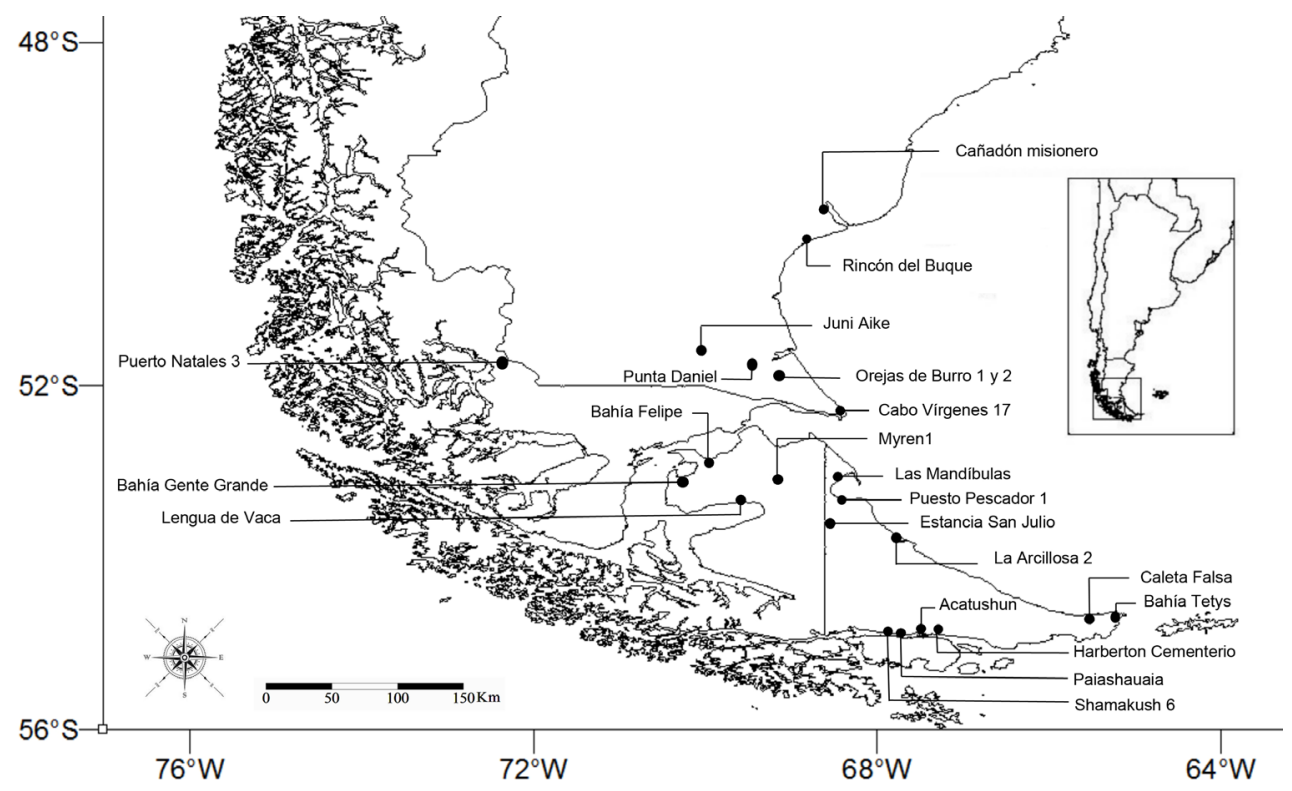

Fig. 2. Ubicación de los sitios arqueológicos analizados en Patagonia Austral.

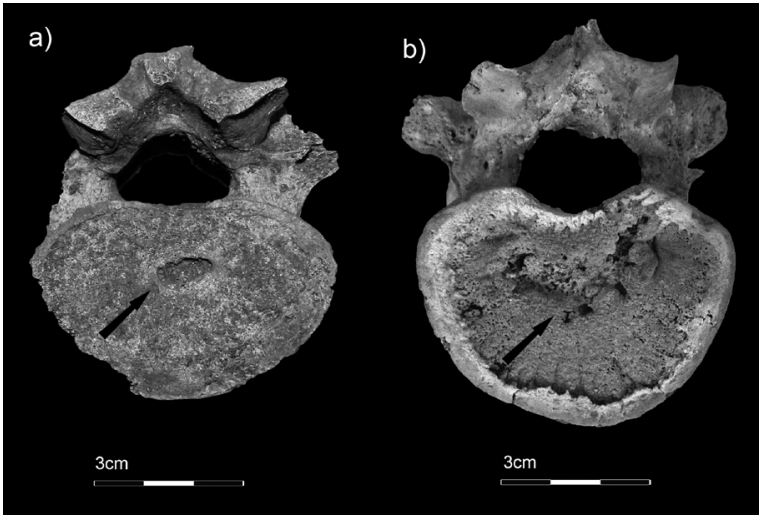

Fig. 3. Nodulos de Schmorl leve (a) y severo (b), según la clasificación de Knüsel et al. (1997).

como 2 (severo) a las lesiones que exceden estos valores (Fig. 3).

Se analizó además la presencia y severidad de osteofitosis vertebrales en los individuos incluidos, con el objetivo de analizar su posible influencia en la formación de NS. Se interpretó como osteofitos a la proliferación de hueso nuevo alrededor de las márgenes articulares de los cuerpos vertebrales (Rogers \& Waldron 1995; Waldron 2009), considerando tres grados de severidad: 1=leve, 2=moderado y 3=severo (Lovell 1994; Rojas-Sepúlveda et al. 2008).

El test de Chi cuadrado fue aplicado para determinar la significancia en las diferencias de las prevalencias de NS entre regiones de origen y dietas de los individuos analizados, empleando una significancia estadística definida por una probabilidad de $p<0,05$. Los datos fueron analizados utilizando el software estadístico Statistica 7.0.

\section{RESULTADOS}

En la tabla 2 se presentan los esqueletos humanos estudiados, mostrándose para cada uno la presencia y número de NS, las vértebras afectadas, su severidad y ubicación. En total fueron registrados 28 NS, afectando a 6 individuos $(27,3 \%)$ de los 22 estudiados, de los cuales 4 (67\%) presentaron lesiones severas, $1(16,6 \%)$ mostró lesiones leves, y 1 individuo $(16,6 \%)$ presentó ambos tipos de lesiones (Tabla 2). De entre los individuos afectados, $2(33,4 \%)$ presentaron una única lesión, mientras los restantes $4(66,6 \%)$ mostraron entre 5 y 8 NS, afectando entre el $4,2 \%$ y el $25 \%$ de las vértebras analizadas en cada individuo. Se observó además una mayor prevalencia de NS en la superficie inferior $(60,7 \%)$ respecto de la superficie superior $(39,2 \%)$ de los cuerpos vertebrales. De acuerdo a la cronología, 4 de los $11(36,4 \%)$ individuos correspondientes a momentos previos al contacto 
Tabla 2. Distribución, severidad y ubicación de los NS en la muestra estudiada. Ref: VNS: vértebras con NS; VO: vértebras observadas; I: inferior; S: superior; T: toráxica; L: lumbar.

\begin{tabular}{|c|c|c|c|c|c|c|c|c|}
\hline Región & Sitio & VNS & $\mathrm{VO}$ & $\%$ & Nro. NS & Vértebras afectadas & Severidad & Ubicación \\
\hline \multirow{4}{*}{$\begin{array}{l}\text { Santa Cruz- } \\
\text { Magallanes }\end{array}$} & Juni Aike & 4 & 23 & 17,4 & 8 & S/IL1, S/IL2, S/IL3, S/IL4 & $1-2$ & central \\
\hline & Puerto Natales 3 & 0 & 18 & 0 & 0 & - & - & - \\
\hline & Cañadón Misionero & 0 & 22 & 0 & 0 & - & - & - \\
\hline & Rincón del Buque & 6 & 24 & 25 & 6 & $\begin{array}{c}\text { IT7, IT8, IT9, IT10, IT11, } \\
\text { IT12 }\end{array}$ & 2 & central \\
\hline \multirow{4}{*}{$\mathrm{n}=8$} & Cabo Vírgenes 17 & 1 & 18 & 5,6 & 1 & IL4 & 1 & central \\
\hline & Punta Daniel & 0 & 23 & 0 & 0 & - & - & - \\
\hline & Orejas de Burro 1 & 5 & 24 & 20,8 & 7 & IT5; IL1, S/IL2, S/IL3, SL4 & 2 & central \\
\hline & Orejas de Burro 2 & 3 & 24 & 12,5 & 5 & S/IL2, S/IL3 y SL5 & 2 & central \\
\hline \multirow{8}{*}{ Norte TdF } & Las Mandíbulas & 0 & 24 & 0 & 0 & - & - & - \\
\hline & Bahía gente grande & 0 & 10 & 0 & 0 & - & - & - \\
\hline & Lengua de Vaca & 0 & 24 & 0 & 0 & - & - & - \\
\hline & Puesto Pescador 1 & 1 & 24 & 4.2 & 1 & SL3 & 1 & central \\
\hline & Estancia San Julio & 0 & 24 & 0 & 0 & - & - & - \\
\hline & Myren 1 & 0 & 16 & 0 & 0 & - & - & - \\
\hline & Bahía Felipe 1 & 0 & 24 & 0 & 0 & - & - & - \\
\hline & La Arcillosa 2 & 0 & 24 & 0 & 0 & - & - & - \\
\hline \multirow{6}{*}{ Sur TdF } & Acatushun & 0 & 21 & 0 & 0 & - & - & - \\
\hline & $\begin{array}{l}\text { Harberton } \\
\text { Cementerio }\end{array}$ & 0 & 23 & 0 & 0 & - & - & - \\
\hline & Bahia Tethys & 0 & 14 & 0 & 0 & - & - & - \\
\hline & Caleta Falsa 1 sitio 8 & 0 & 20 & 0 & 0 & - & - & - \\
\hline & Paiashauaia & 0 & 23 & 0 & 0 & - & - & - \\
\hline & Shamakush 6 & 0 & 22 & 0 & 0 & - & - & - \\
\hline
\end{tabular}

presentaron NS, mientras que 2 de los $11(18,2 \%)$ esqueletos de momentos durante el contacto estuvieron afectados.

Todos los NS fueron registrados en las vértebras dorsales y lumbares, afectando principalmente el segmento T7-L4, aunque uno de los individuos (Rincón del Buque) presentó NS sólo en las vértebras dorsales. Todas las lesiones se ubican en la región central de los cuerpos vertebrales. Además, en todos los casos afectaron a individuos masculinos. Respecto de la edad de los individuos afectados, $4(66,6 \%)$ poseen edades entre 20-35 años, mientras los 2 restantes (33,3\%) corresponden a individuos que murieron entre 3549 años. Los individuos que presentaron un único NS corresponden al grupo más joven, mientras los individuos con múltiples NS poseen edades que se incluyen tanto entre los adultos jóvenes como entre los adultos medios.
Cuando se evaluó la variabilidad de la presencia de NS según la región de Patagonia Austral, se observó que 27 de los 28 NS $(96,4 \%)$ corresponden a individuos recuperados en la región de Santa Cruz/Magallanes, mientras la restante lesión fue identificada en un individuo del norte de Tierra Fuego (Tabla 3). En este sentido, 5 de los 6 individuos afectados (83,3\%) pertenecen a la región continental (Santa Cruz/Magallanes). En esta región, 5 de los 8 individuos evaluados $(62,5 \%)$ presentaron NS (Tabla 3$)$, de los cuales 4 (80\%) mostraron entre 5 y 8 NS (Tabla 2).

Debido a la mayor prevalencia de NS en la región de Santa Cruz/Magallanes, se estudió la posible relación de su desarrollo con la presencia de osteofitosis vertebrales. En la Tabla 4 se muestran los porcentajes de osteofitosis marginales por región de Patagonia Austral. Se observó que este tipo de lesiones degenerativas articulares afectó 
Tabla 3. Número de NS e individuos afectados entre el total de esqueletos de Patagonia Austral evaluados, y clasificados por región y dieta. Ref: * $\chi 2$ significativamente diferente a las otras dos condiciones evaluadas, con $\mathrm{p}<0,05$.

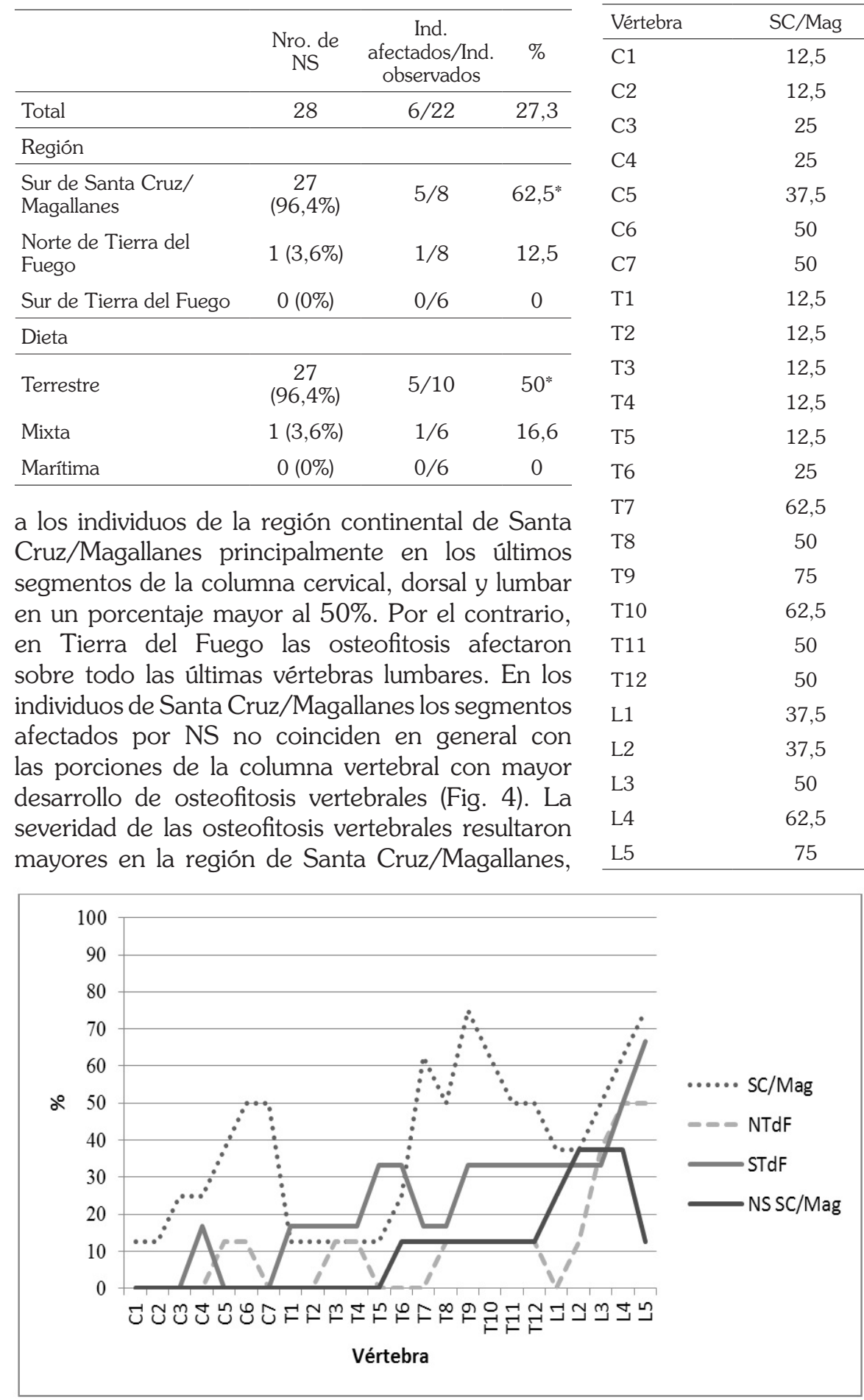

Tabla 4. Porcentajes de vértebras afectadas con osteofitos marginales clasificadas por regiones en Patagonia Austral. Ref: SC/Mag: Santa Cruz/Magallanes; NTdF: Norte de Tierra del Fuego; STdF: Sur de Tierra del Fuego.

\begin{tabular}{|c|c|c|c|}
\hline Vértebra & SC/Mag & $\mathrm{NTdF}$ & STdF \\
\hline $\mathrm{C} 1$ & 12,5 & 0 & 0 \\
\hline $\mathrm{C} 2$ & 12,5 & 0 & 0 \\
\hline $\mathrm{C} 3$ & 25 & 0 & 0 \\
\hline $\mathrm{C} 4$ & 25 & 0 & 16,7 \\
\hline C5 & 37,5 & 12,5 & 0 \\
\hline C6 & 50 & 12,5 & 0 \\
\hline C7 & 50 & 0 & 0 \\
\hline $\mathrm{T} 1$ & 12,5 & 0 & 16,7 \\
\hline $\mathrm{T} 2$ & 12,5 & 0 & 16,7 \\
\hline T3 & 12,5 & 12,5 & 16,7 \\
\hline $\mathrm{T} 4$ & 12,5 & 12,5 & 16,7 \\
\hline T5 & 12,5 & 0 & 33,3 \\
\hline T6 & 25 & 0 & 33,3 \\
\hline $\mathrm{T} 7$ & 62,5 & 0 & 16,7 \\
\hline T8 & 50 & 12,5 & 16,7 \\
\hline T9 & 75 & 12,5 & 33,3 \\
\hline T10 & 62,5 & 12,5 & 33,3 \\
\hline T11 & 50 & 12,5 & 33,3 \\
\hline T12 & 50 & 12,5 & 33,3 \\
\hline L1 & 37,5 & 0 & 33,3 \\
\hline L2 & 37,5 & 12,5 & 33,3 \\
\hline L3 & 50 & 37,5 & 33,3 \\
\hline L4 & 62,5 & 50 & 50,0 \\
\hline L5 & 75 & 50 & 66,7 \\
\hline
\end{tabular}

Fig.4. Porcentaje de osteofitosis por vertebras en individuos de Patagonia Austral, clasificados según la región de origen. Ref: SC/ Mag: Santa Cruz/Magallanes; NTdF: Norte de Tierra del Fuego; STdF: Sur de Tierra del Fuego; NS SC/Mag: nódulos de Schmorl en Santa Cruz/Magallanes. 
Fig.5. Porcentaje de grado de severidad de las osteofitosis registradas en los individuos de Patagonia Austral, clasificadas por regiones.

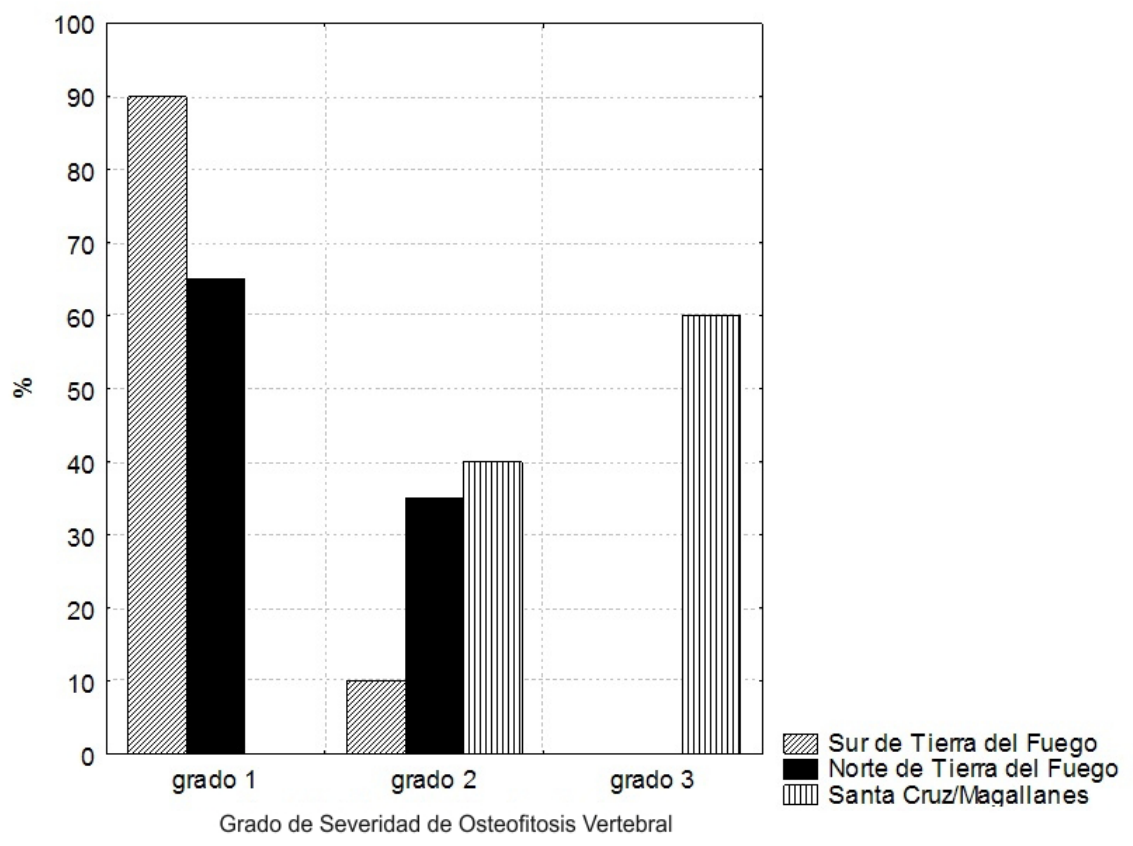

cuyos individuos mostraron osteofitosis de grado 2 y 3, mientras en el norte y sur de Tierra del Fuego presentaron lesiones de grado 1 y 2 (Fig. 5).

\section{DISCUSIÓN Y CONCLUSIONES}

Los NS son lesiones vertebrales frecuentemente halladas en poblaciones modernas y antiguas. A pesar de su ubicuidad, su etiología y consecuencias son sólo parcialmente comprendidas. Uno de los aspectos más variables de los NS es su prevalencia, la cual ha mostrado amplias diferencias interpoblacionales, ya sea en estudios actuales como arqueológicos, probablemente influidas por los criterios metodológicos y la naturaleza de las muestras, así como por factores biológicos y culturales. Los resultados obtenidos en este trabajo muestran una prevalencia de NS de $27,3 \%$ en restos de Patagonia Austral, que resulta similar a las reportadas en otras muestras esqueletales (Fabra et al. 2012; Novak \& Šlaus 2011; Üstündağ 2009; Woo \& Pak 2012), aunque menor a las observadas en algunos estudios clínicos, las cuales pueden alcanzar hasta el 75\% (Faccia \& Williams 2008; Pfirrmann \& Resnick 2001).

En la muestra estudiada en este trabajo se observó una marcada tendencia geográfica, con una prevalencia significativamente mayor en los individuos de la región de Santa Cruz/Magallanes con respecto a los de las regiones norte y sur de Tierra del Fuego. Debido al reducido número de individuos analizados no es posible descartar sesgos muestrales como causa de estas tendencias, aunque la variabilidad espacial podría también ser explicada por diferencias poblacionales biológicas y en las prácticas culturales reportadas.

La diversidad biológica entre poblaciones podría ser una de las causas de la variabilidad espacial en la prevalencia de NS en Patagonia Austral. Sobre este aspecto, Dar et al. (2010) indican que el desarrollo de los NS podría estar influido por el origen étnico. Estudios basados en ADN mitocondrial sugieren que los grupos de la región meridional de Santa Cruz y los grupos de Tierra del Fuego comparten ancestros comunes (Lalueza et al. 1997), mientras que análisis de la variabilidad craneofacial tienden a agrupar las poblaciones fueguinas por un lado y las continentales por otro (Cocilovo \& Guichón, 1985-86; González-José et al. 2001). Desde un punto de vista etnográfico, principalmente dos grupos ocuparon el sur de Patagonia y Tierra del Fuego: 1) cazadores recolectores terrestres (Tehuelches/Aonikenk en el continente y Selk'nam 
en el norte de Tierra del Fuego), tradicionalmente descriptos como cazadores de guanacos; y 2) cazadores-recolectores marítimos (Kaweskar y Yamanas en el litoral sur de Tierra del Fuego), especializados en la explotación de recursos marinos (e.g. Gusinde, 1982, 1986; Hyades \& Deniker 1891; ver además Morello et al. 2012; Orquera \& Piana 2009). Sin embargo, evidencias arqueológicas y paleodietarias sugieren escenarios más complejos y no completamente coincidentes con las clasificaciones etnográficas (e.g. Yesner et al., 2003), las cuales resultan difíciles de sostener para periodos temporales amplios y sólo defendible para momentos históricos (Borrero 2001).

En este sentido, Plomp et al. (2012) propusieron que la forma y morfología normal de las últimas vértebras dorsales, variable entre poblaciones debido en parte a su constitución biológica, tendría un efecto relevante en la formación de los NS debido a los movimientos de flexión, extensión y rotación a los cuales está sometida esta porción de la columna dorsolumbar y al menor espesor de los discos intervertebrales en relación con el tamaño de los cuerpos vertebrales. En esta muestra particular de Patagonia Austral las lesiones afectaron la región inferior de la columna dorsal y superior de la columna lumbar, resultados que acompañan la tendencia general reportada en estudios clínicos y bioarqueológicos (Peng et al. 2003; Pfirrmann \& Resnick 2001; Üstündağ 2009), y que podrían coincidir con la causas morfológicas asociadas a este segmento de la columna vertebral. Podría interpretarse entonces que la mayor prevalencia de NS correspondería a los individuos que ocuparon territorios asignables etnográficamente a la etnia Tehuelche, y que podrían tener una mayor susceptibilidad al desarrollo de NS. Sin embargo, no existe información disponible acerca de aspectos morfológicos vertebrales que sugieran diferencias entre poblaciones continentales y fueguinas que pudieran relacionarse con la presencia de NS, por lo que por el momento no es posible sostener o descartar que las poblaciones de Santa Cruz/Magallanes tuvieran una mayor predisposición biológica a generar este tipo de lesiones vertebrales.

Como consecuencia de la asociación con aspectos morfológicos vertebrales normales, Plomp et al. (2012) indican que la interpretación de los NS como indicadores de actividad física debe ser tomada con cautela. Sin embargo, una parte importante de los resultados alcanzados en restos esqueletales atribuyen la presencia de NS al estrés mecánico y los traumas repetitivos relacionados con patrones de actividad física producto de prácticas económicas y culturales (Knüsel et al. 1997; Lai \& Lovell 1992; Novak \& Salus 2011; Šlaus 2000; Üstündağ et al. 2009). De acuerdo con los resultados presentados en este estudio las prácticas culturales y la actividad física asociadas a economías basadas predominantemente en recursos terrestres descriptas para las poblaciones de Patagonia Austral podrían estar vinculadas a un mayor desarrollo de NS, dado que individuos con este tipo de economía presentaron prevalencias significativamente mayores que los individuos con dietas mixtas o con mayor aporte de recursos marítimos. Sin embargo, sólo se encontró una mayor frecuencia en los individuos provenientes de la región continental con este tipo de dieta.

Los individuos de Santa Cruz/Magallanes presentaron además un desarrollo de osteofitosis vertebrales de mayor evolución que en los individuos del norte y sur de Tierra del Fuego, lo que podría explicar la variabilidad espacial en el desarrollo de NS respecto de los individuos del norte de Tierra del Fuego. Los análisis originales acerca de la formación de NS (Schmorl \& Junghanns 1971:166) sugieren que estarían asociados a cambios degenerativos de las vértebras producidos al estrés mecánico por actividad física. Estudios clínicos y paleopatológicos más recientes presentan resultados poco concluyentes al respecto (e.g. González-Reimers et al. 2002; Hilton et al. 1976; Pfirrmann \& Resnick 2001), aunque parecen coincidir en que los NS podrían vincularse a cambios degenerativos de moderada evolución (Pfirrmann \& Resnick 2001). En este trabajo el patrón de distribución de los osteofitos y los NS sobre la columna vertebral no coinciden completamente, dado que los primeros afectaron en mayor medida la porción final de la columna cervical, la porción media de la columna dorsal y las últimas vertebras de la columna lumbar, mientras los NS se identificaron sobre todo en la porción final de la columna dorsal y las primeras vértebras lumbares. Los osteofitos vertebrales, por lo tanto, no parecen contribuir directamente en la 
formación de NS. Sin embargo, el mayor grado de severidad de la osteofitosis en los esqueletos de Santa Cruz/Magallanes podría indicar actividades físicas de mayor magnitud, con efectos sobre la columna vertebral que podrían favorecer a su vez el desarrollo de hernias de los discos vertebrales.

La mayoría de los individuos presentan múltiples NS, lo que permitiría defender las causas mecánicas como la etiología que mejor explica este tipo de lesiones. Según Pfirrmann \& Resnick (2001) los NS producidos por lesiones traumáticas suelen ser aislados y ubicarse en la parte posterior del platillo vertebral y en las últimas vertebras dorsales, a diferencia de lo que se observa aquí, ubicados siempre en el centro de los cuerpos vertebrales afectando además en mayor proporción a las vértebras lumbares, coincidiendo con los resultados reportados por Dar et al. (2010).

Considerando la cronología, los NS afectaron tanto a individuos de momentos previos como durante el contacto. En este sentido, los cambios culturales propuestos para el periodo de proceso de contacto aborigen-europeo (e.g. Guichón 1994; Guichón et al. 2006) parecen haber producido una disminución en el desarrollo de este tipo de lesiones patológicas. Sin embargo, el reducido tamaño de la muestra estudiada requiere futuras evaluaciones sobre este aspecto, analizando la prevalencia en escalas temporales más acotadas.

Los NS afectaron tanto individuos adultos jóvenes como adultos medios, por lo que la edad no parece ser un causante de NS en estos individuos. De igual manera, Saluja et al. (1986) y Üstündağ (2009) no encontraron en sus estudios asociación entre los NS y el aumento de la edad. Sin embargo, las causas de la presencia de estas lesiones en ambos grupos etarios podrían no ser semejantes, dado que se ha propuesto que en individuos jóvenes la ocurrencia de traumas o la presencia de áreas debilitadas en los platillos vertebrales parece ser las principales causas de la formación de NS, mientras en grupos de adultos mayores son los cambios degenerativos los que contribuyen a su formación (Kerr \& Resnick 1984; Pfirrmann \& Resnick 2001).

Todos los esqueletos afectados corresponden a individuos masculinos, aunque sólo unos pocos individuos femeninos fueron incluidos en este estudio, por lo que el sesgo muestral no puede ser descartado como posible explicación de las diferencias sexuales. La asociación de los NS con el sexo no es completamente clara. Mientras algunos autores sostienen que no existe una relación directa de este tipo de lesiones con el sexo (Pfirrmann \& Resnick 2001; Dar et al. 2010), mayores prevalencia de NS a favor de los individuos masculinos fueron reportadas en otros trabajos, atribuidas en general a mayores influencias mecánicas asociadas a la actividad física, a traumas asociados a la actividad (Faccia \& Willilams 2008; Šlaus 2000; Üstündağ 2009) y al mayor tamaño de los cuerpos vertebrales en los hombres (Plomp et al. 2012).

Teniendo en cuenta los resultados obtenidos y su interpretación en base a la información clínica y bioarqueológica previa, la conjugación de posibles factores biológicos y la actividad física asociada a las prácticas económicas cazadoras-recolectoras terrestres podría haber favorecido la formación de NS en los individuos de la región continental de Patagonia. En contraste, individuos con el mismo tipo de economía en Tierra del Fuego muestran menores prevalencias de esta patología vertebral. En tal sentido, no puede descartarse la influencia de diferencias biológicas, tanto en el desarrollo como en la morfología vertebral, como causa de una mayor susceptibilidad a la formación de NS en las poblaciones continentales respecto de las fueguinas.

$\mathrm{Al}$ igual que en otras lesiones degenerativas, la interacción entre factores culturales y biológicos aún resulta difícil de esclarecer en la formación de los NS, por lo que el análisis de restos esqueletales cobran relevancia para la interpretación de información proveniente de estudios sobre poblaciones actuales y antiguas. En el caso del estudio de la salud de las poblaciones del sur de Patagonia, investigaciones futuras acerca del desarrollo de lesiones articulares y cambios entésicos, tanto en el esqueleto axial como apendicular, permitirán fortalecer las conclusiones halladas en este trabajo.

\section{AGRADECIMIENTOS}

Al Dr. Gustavo Flensborg por sus valiosos comentarios sobre el manuscrito. A la Mag. Susana Morano (Instituto de la Patagonia, Punta 
Arenas, Chile) y la Lic. Claudia Aranda (Museo Etnografico J.B. Ambrosetti, Buenos Aires) por su colaboración y acceso a los restos conservados en esas instituciones. A los Dres. Luis Borrero, Ricardo Guichón, Mónica Salemme, Fernando Santiago, Ernesto Piana y Francisco Zangrando por el acceso a los restos recuperados en el marco de sus respectivos proyectos de investigación. A dos revisores anónimos, cuyos comentarios contribuyeron a mejorar este trabajo. Los estudios fueron realizados con el financiamiento del Proyecto ANPCyT - PICT-0385-2008.

\section{BIBLIOGRAFÍA}

Barnes, E. (2005). Diseases and Human Evolution. Albuquerque: University of New Mexico Press.

Bass, W. M. (1995). Human osteology. A laboratory and field manual of the human skeleton. Columbia: Missouri Archaeological Society

Borrero, L. A. (2001). El poblamiento de la Patagonia. Toldos, milodones y volcanes. Buenos Aires: Emecé.

Borrero, L. A. \& Barberena, R. (2006). Hunter-gatherer home ranges and marine resources. An archaeological case from Southern Patagonia. Current Anthropology, 47(5), 855-867.

Brooks, S. T. \& Suchey, J. M. (1990). Skeletal age determinations based on the Os Pubis: A comparison of the AcsádiNemeskéri and Suchey-Brooks Methods. Human Evolution, 5, 227-238.

Buikstra, J. E. \& Ubelaker, D. H. (1994). Standars for data collection from human skeletal remains. Arkansas: Arkansas Archaeological Survey Research Series $\mathrm{N}^{\circ} 44$.

Chandraraj, S., Briggs, C. A. \& Opeskin, K. (1998). Disc herniation in the young and end-plate vascularity. Clinical Anatomy, 11, 171-176.

Cocilovo, J. A. \& Guichón, R. A. (1985-1986). Propuesta para el estudio de las poblaciones aborígenes del extremo austral de Patagonia. Anales del Instituto de la Patagonia (Serie Ciencias Sociales), 6, 111-123.

Dar, G., Masharawi ,Y., Peleg, S., Steinberg, N., May, H., Medlej, B., Peled, N. \& Hershkovitz, I. (2010). Schmorl's nodes distribution in the human spine and its possible etiology. European Spine Journal, 19, 670-675.

Fabra, M., González C. V. \& Salega M. S. (2012). Modos de vida e historia biológica de poblaciones de las sierras y las llanuras de Córdoba (Argentina): aproximaciones desde el registro bioarqueológico. Revista Argentina de Antropología Biológica 14(Número Especial), 87-104.

Faccia, K. J. \& Williams R. C. (2008). Schmorl's nodes: clinical significance and implications for the bioarcheological record. International Journal of Osteoarchaeology, 18, 28-44.

Fahey, V., Silberstein, M., Anderson, R. \& Briggs, C. (1998). The pathogenesis of Schmorl's nodes in relation to acute trauma: an autopsy study. Spine, 23, 22722275.

González-José, R., Dahinten, S. L., Luis, M. A., Hernández, M. \& Pucciarelli H. M. (2001). Craneometric variation and the settlement of the Americas: testing hypotheses by means of R-matrix and matrix correlation analyses. American Journal of Physical Anthropology, 116, 154-165.

González-Reimers, E., Mas-Pascual, M., Arnay-de-la-Rosa, M., Velasco-Vázquez, J. \& Santolaria-Fernández. F. (2002). Schmorl nodes: lack of relationship between degenerative changes and osteopenia. Radiology, 222, 293-294.

Guichón, R. A. (1994). Antropología física de Tierra del Fuego, caracterización biológica de las poblaciones prehispánicas. Tesis doctorado, Facultad de Filosofía y Letras de la Universidad de Buenos Aires, Buenos Aires.

Guichón, R. A. \& Suby, J. A. (2011). Estudio bioarqueológico de los restos humanos recuperados por Anne Chapman en Caleta Falsa, Tierra del Fuego. Magallania, 39(1), 163-177.

Guichón, R. A., Muñoz, A. S. \& Borrero, L. A. (2000). Datos para una tafonomía de restos óseos humanos en Bahía San Sebastián, Tierra del Fuego. Relaciones de la Sociedad Argentina de Antropología, XXV, 297-313.

Guichón, R. A., Suby, J. A., Casali, R. \& Fugassa, M. (2006). Health at the time of native-european contact in Southern Patagonia. Memorias do Instituto Oswaldo Cruz, 101(Suppl. II), 97-105.

Gusinde, M. (1982). Los Indios de Tierra del Fuego. Los Selk'nam. Buenos Aires: Centro Argentino de Etnología Americana.

Gusinde, M. (1986). Los Indios de Tierra del Fuego. Los Yamana. Buenos Aires: Centro Argentino de Etnología Americana.

Haidar, R., Ghanem, I., Saad, S. \& Uthman, I. (2009). Lumbar disc herniation in young children. Acta Pæediatrica, 99, 19-23. 
Hilton, R. C., Ball, J. \& Benn, R. T. (1976). Vertebral end-plate lesions (Schmorl's nodes) in the dorsolumbar spine. Annals of the Rheumatic Diseases, 35, 127-132.

Hyades, P. \& Deniker, J. (1891). Anthropologie et ethnographie. Mission scientifique du Cap Horn, 18821883. Paris: Gauthier-Villars et fils.

Kerr, R. \& Resnick, D. (1984). Degenerative diseases of the spine. Australasian Radiology, 28, 319-329.

Knüsel, C. J., Gögel, S. \& Lucy, D. (1997). Comparative degenerative joint disease of the vertebral column in the medieval monastic cemetery of the Gilbertine Priory of St. Andrew, Fishergate, York, England. American Journal of Physical Anthropology, 103, 481-495.

Lai, P. \& Lovell, N. C. (1992). Skeletal markers of occupational stress in the fur trade: a case study from a Hudson's Bay company fur trade post. International Journal of Osteoarchaeology, 2, 221-234.

Lalueza, C., Perez-Perez, A., Prats, E., Cornudella, L. \& Turbon, D. (1997). Lack of founding amerindian mitochondrial DNA lineages in extinct aborigines from Tierra de Fuego-Patagonia. Human Molecular Genetics, 6, 41-46.

L'Heureux, G. L. \& Barberena, R. (2008). Evidencias bioarqueológicas en Patagonia meridional: el sitio Orejas de Burro 1 (Pali Aike, provincia de Santa Cruz). Intersecciones en Antropología, 9, 11-24.

L’Heureux, G., Guichón, R. A., Barberena, R. \& Borrero, L. A. (2003). Durmiendo bajo el faro. Estudio de un entierro humano en Cabo Vírgenes (C.V.17), Pcia. De Santa Cruz, República Argentina. Intersecciones en Antropología, 4, 87-98.

Lovell, N. C. (1994). Spinal arthritis and physical stress at Bronze Age Harappa. American Journal of Physical Anthropology, 93, 149-164.

McNaught, J. M. (2006). A clinical and archaeological study of Schmorl's Nodes: using clinical data to understand the past. (Tesis de Doctorado, Durham University). Recuperado de Durham E-Theses Online: http:// etheses.dur.ac.uk/2689/

Moore, R. J. (2006). The vertebral endplate: disc degeneration, disc regeneration. European Spine Journal, 15 (Suppl. 3), S333-S337.

Morello, F., Borrero, L., Massone, M., Stern, C., GarcíaHerbst, A., McCulloch, R., Arroyo-Kalin, M., Calás, E., Torres, J. \& Prieto, A. (2012). Hunter-gatherers, biogeographic barriers and the development of human settlement in Tierra del Fuego. Antiquity, 86, 71-87.
Novak, M. \& Šlaus, M. (2011). Vertebral pathologies in two early modern period (16th-19th century) populations from Croatia. American Journal of Physical Anthropology 145,270-281.

Peng, B., Wu, W., Hou, S., Shang, W., Wang, X. \& Yang, Y. (2003). The pathogenesis of Schmorl's nodes. Journal of Bone and Joint Surgery, 85, 879-882.

Pfirrmann, C. W. \& Resnick, D. (2001). Schmorl nodes of the thoracic and lumbar spine: radiographic-pathologic study of prevalence, characterization, and correlation with degenerative changes of 1, 650 spinal levels in 100 cadavers. Radiology, 219, 368-374.

Orquera, L. \& Piana, E. (2009). Sea nomads of the Beagle Channel in Southernmost South America: over six thousand years of coastal adaptation and stability. The Journal of Island and Coastal Archaeology, 4(1), 61-81.

Piana, E., Tessone, A. \& Zangrando, A. F. (2006). Contextos mortuorios en la región del canal Beagle... del hallazgo fortuito a la búsqueda sistemática. Magallania, 34(1), 103-117.

Plomp, K. A., Roberts, C. A. \& Viðarsdóttir, U. S. (2012). Vertebral morphology influences the development of Schmorl's nodes in the lower thoracic vertebrae. American Journal of Physical Anthropology, 149, 572-582.

Resnick, D. \& Niwayama, G. (1978). Intravertebral disk herniations: cartilaginous (Schmorl's) nodes. Radiology, 126, 57-65.

Robb, J., Bigazzi, R., Lazzarini, L., Scarsini, C., \& Sonego F. (2001). Social "status" and biological "status": a comparison of grave goods and skeletal indicators from Pontecagnano. American Journal of Physical Anthropology, 115, 213-222.

Roberts, S., Menage, J. \& Urban, J. P. (1989). Biochemical and structural properties of the cartilage end-plate and its relation to the intervertebral disc. Spine, 14, 166-174.

Rogers, J. \& Waldron, T. (1995). A Field Guide to Joint Diseases in Archaeology. Chichester: John Wiley and Sons.

Rojas-Sepúlveda, C., Ardagna, Y. \& Dutour, O. (2008). Paleoepidemiology of vertebral degenerative disease in a pre-Columbian Muisca Series from Colombia. American Journal of Physical Anthropology, 135, 416-430.

Saluja, G., Fitzpatrick, K., Bruce, M. \& Cross, J. (1986). Schmorl's nodes (intervertebral herniations of intervertebral disc tissue) in two historic British 
populations. Journal of Anatomy, 145, 87-96.

Santiago, F., Salemme, M., Suby, J. A. \& Guichón R. A. (2011). Restos óseos humanos en el norte de Tierra del Fuego. Aspectos contextuales, dietarios y paleopatológicos. Intersecciones en Antropología, 12, 147-162.

Schinder, G. \& R.A. Guichón. (2003). Isótopos estables y estilo de vida en muestras óseas humanas de Tierra del Fuego. Magallania, 31, 33-44.

Schmorl, G. \& Junghanns, H. (1971). The Human Spine in Health and Disease. Second American edition. New York: Grune and Stratton.

Šlaus, M. (2000). Biocultural analysis of sex differences in mortality profiles and stress levels in the late medieval population from Nova Raca, Croatia. American Journal of Physical Anthropology, 111, 193-209.

Suby, J. A. \& Guichón, R. A. (2010). Los restos óseos humanos de la colección de la misión "La Candelaria" (Rio Grande, Tierra del Fuego). Magallania, 38(2), 121133.

Suby, J.A., Salemme, M. \& Santiago, F. (2008). Análisis paleopatológico de los restos humanos del sitio Puesto Pescador 1 (Tierra del Fuego). Magallania, 36(1), 53-64.

Suby, J. A., Guichón R. A. \& Zangrando, A. F. (2009). El registro biológico humano de la costa meridional de Santa Cruz. Revista Argentina de Antropología Biológica, 11(1), 109-124.

Takahashi, K, Miyazaki, T., Ohnari, H., Takino, T. \& Tomita, K. (1995). Schmorl's nodes and low-back pain: analysis of magnetic resonance imaging find- ings in symptomatic and asymptomatic individuals. European Spine Journal, 4, 56-59.

Tessone, A., Guichón, R. A., Suby, J. A. \& Kozameh, L. (2011). Bioarqueología de Península Mitre. En Zangrando, F., Vázquez, M. \& Tessone, A. (Eds), "Los cazadores-recolectores del extremo oriental fueguino. Arqueología de Península Mitre e Isla de los Estados (pp. 231-270). Buenos Aires: Sociedad Argentina de Antropología.

Üstündağ, H. (2009). Schmorl's nodes in a post-medieval skeletal sample from Klostermarienberg, Austria. International Journal of Osteoarchaeology, 19, 695-710.

Wagner, A. L, Murtagh, F. R., Arrington, J. A. \& Stallworth, D. (2000). Relationships of Schmorl's nodes to vertebral body endplate fractures and acute endplate disk extrusions. American Journal of Neuroradiology, 21, 276-281.

Waldron, T. (2009). Paleopathology. Cambridge: Cambridge University Press.

Weiss, E. (2005). Schmorl's nodes: a preliminary investigation. Paleopathology Association Newsletter, 132, 6-10.

Williams, F. M. K., Manek, N. J., Sambrook, P. N., Spector, T. D. \& MacGregor, A. J. (2007). Schmorl's Nodes: common, highly heritable, and related to lumbar disc disease. Arthritis \& Rheumatism (Arthritis Care \& Research), 57(5), 855-860.

Woo, E. J. \& Pak, S. (2012). The relationship between the two types of vertebral degenerative joint disease in a Joseon Dynasty population, Korea. International Journal of Osteoarchaeology, DOI: 10.1002/ oa. 2250 .

Yasuma, T., Saitd, S. \& Kihara, K. (1988). Schmorl's Nodes. Correlation of X-ray and histological findings in postmortem specimens. Acta Pathological Japonica, 38(6), 723-733.

Yesner, D. R., Figuerero Torres, M. J., Guichón, R. A \& Borrero, L. A. (2003). Stable isotope analysis of human bone and ethnohistoric subsistence patterns in Tierra del Fuego. Journal of Anthropological Archaeology, 22, 279-291.

Zhang, N., Li, F. C., Huang, Y-J., Teng, C. \& Chen, W-S. (2010). Possible key role of immune system in Schmorl's nodes. Medical Hypotheses, 74, 552554. 
